\title{
Diagnosis and Endovascular Management of Segmental Heel Ischemia
}

\author{
Miguel Montero-Baker ${ }^{1 *}$, Bernadino Castelo Branco Rocha ${ }^{2}$, David G Armstrong ${ }^{1}$, Kay R Goshima ${ }^{1}$, \\ John Miller ${ }^{1}$ and Joseph L Mills Sr \\ ${ }^{1}$ Division of Vascular and Endovascular Surgery, University of Arizona Health Sciences Center, Tucson, \\ Arizona, USA \\ ${ }^{2}$ Division of Surgery, University of Arizona Health Sciences Center, Tucson, Arizona, USA

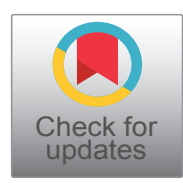

*Corresponding author: Miguel Montero-Baker, Division of Vascular and Endovascular Surgery, University of Arizona Health Sciences Center, 1501 N Campbell Ave, PO Box: 245072, Tucson, Arizona, USA, Tel: 85724-5072, E-mail: montero. bkr@gmail.com

\begin{abstract}
Objective: (1) Describe a very specific subgroup of patients that we have termed Orphan Heel Syndrome (OHS) characterized by the clinical triad of: Diabetic chronic kidney disease (CKD), posterior tibial and peroneal arterial occlusive disease and an ischemic heel ulcer; and (2) Analyze the applicability of endovascular therapy and its clinical outcomes in a well-delineated cohort of OHS patients.
\end{abstract}

Methods: Retrospective review of 40 consecutive patients treated at the University of Arizona for full thickness diabetic heel ulcers requiring debridement between 1 January 2009 and 31 December 2011.

Results: Over the 3-year study period, among a consecutive series of 40 diabetic heel ulcers, 12 patients (14 limbs) with typical clinical and angiographic findings of OHS were identified. The mean age of this subgroup was $68.3 \pm 12.8$ years and $75 \%(n=9 / 12)$ were male. All patients had poorly controlled diabetes with a mean hemoglobin A1C of $9.4 \pm 0.9 \mathrm{mg} / \mathrm{dl}$ and chronic kidney disease (CKD), with $75 \%(n=9 / 12)$ on dialysis. Doppler waveform analysis demonstrated acceptable Anterior Tibial Artery flow, but blunted or non-existing Posterior Tibial Artery flow. Diagnostic angiography confirmed the pattern of severe posterior tibial and peroneal artery occlusive disease. All patients were poor open surgical candidates and were managed with a targeted initial endovascular approach with a technical success rate of $78.6 \%(n=11 / 14)$. At a mean follow-up of 235 days (range $90-956$ ), $50 \%$ of the patients had completely healed their wounds and two patients $(14 \%)$ required major limb amputation at the below knee level.

Conclusion: Orphan heel are difficult to recognize via traditional noninvasive vascular testing and newer techniques to evaluate topographical perfusion warrant investigation. After endovascular-first therapy, limb salvage is acceptable, but wound-healing rates are poor despite application of advanced endovascular therapy.

\begin{abstract}
Keywords
Orphan heel syndrome, Peripheral artery disease, Diabetic foot, Diabetic foot ulcer, Diabetes complications, Chronic kidney disease, Ischemic heel ulcer, Gangrene, Angioplasty, Endovascular therapy
\end{abstract}

\section{Introduction}

Diabetic foot ulcers are common, costly, frequently recurrent and chronic. Once infected they all too often are the precursors of major limb amputation. Increased pressure decreased angiogenic response, neuropathy, trauma and deformity all contribute in varying degrees to the pathogenesis of these ulcers. Patients with limited mobility are at higher risk of developing an even more morbid subtype of diabetic foot complication: The heel ulcer [1]. Authors have reported up to $14 \%$ prevalence of heel ulcers among patients with threatened limbs and severe ischemia requiring vascular intervention $[2,3]$.

Heel ulcers are generally found among a sicker subset of diabetic foot ulcer patients typically characterised by poor ambulatory status, low albumin levels, high rates of gangrene, a need for revascularization with possible major limb amputation and a low 5-year survival. Conservative, non-operative management for this group using total contact casting has been plagued with substandard results [4]. Debridement and free tissue transfer with or without revascularization have been described in a number of publications with mixed results [5-8]. Ad-

Citation: Montero-Baker M, Rocha BCB, Armstrong DG, Goshima KR, Miller J, et al. (2018) Diagnosis and Endovascular Management of Segmental Heel Ischemia. Int J Clin Cardiol 5:117. doi. org/10.23937/2378-2951/1410117

Accepted: June 02, 2018; Published: June 04, 2018

Copyright: (C) 2018 Montero-Baker M, et al. This is an open-access article distributed under the terms of the Creative Commons Attribution License, which permits unrestricted use, distribution, and reproduction in any medium, provided the original author and source are credited.

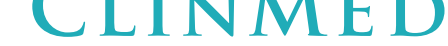

INTERNATIONAL LIBRARY 
ditionally, primary amputation has been advocated for those with end stage renal disease or large heel ulcers $(>4 \mathrm{~cm})[9,10]$.

Previously, we described an angiographic classification system for ischemic heel ulcers and proposed a management algorithm for these patients incorporating wound debridement, open revascularization and tissue transfer [7]. In that study, no patients were managed by endovascular therapy (EVT).

The introduction of EVT has greatly expanded revascularization options for patients with threatened limbs and severe ischemia who are poor surgical candidates. Despite a paradigm shift toward EVT for lower extremity peripheral artery disease (PAD), to date, reports evaluating its role in managing patients with heel ulcers are few in number $[11,12]$.

The purpose of this study is two-fold: (1) To describe a very specific subgroup of patients with a condition that we have termed Orphan Heel Syndrome (OHS). These patients manifest the clinical triad of: Diabetic chronic kidney disease (CKD), a unique pattern of isolated heal circulation with ischemia due to Posterior Tibial and Peroneal Artery occlusive disease (lacking and collateral cross coverage from the Anterior Tibial Artery), and heel ulceration (Figure 1). (2) To examine the applicability and clinical outcomes of endovascular revascularization in $\mathrm{OHS}$ patients.

\section{Methods}

We performed an IRB-approved, retrospective review of all patients who presented to the Southern Arizona Limb Salvage Alliance (SALSA) clinic, at the University of Arizona Medical Center, with complex multifactorial diabetic heel ulcers from 1 January 2009 to 31 December 2011. Waiver of patient consent was authorised given the retrospective nature of our analysis that did not affect patient care or welfare. A total of 40 patients with
42 ulcers were identified. All 42-heel ulcerations were treated with standard of care including: Offloading, regular sharp debridement and clean dressing changes.

Among this cohort, 12 patients with 14 affected limbs underwent diagnostic angiography and targeted endovascular therapy. This subset was evaluated based on a complete diagnostic angiogram with run off that included magnified views of the foot in both antero-posterior and lateral projections. The OHS subgroup under review in this article was distinguished using these detailed, diagnostic angiographic studies. In many cases, due to the very low velocities, prolonged exposure in digital subtraction mode was used to verify the lack of late collateral perfusion of the heel. It is quite possible that more patients in this study were affected by a similar disease pattern and the diagnosis was missed because our recognition and evaluation of these patients evolved during the study period.

Patient variables extracted included: Age, gender, comorbidities, vascular non-invasive studies, angiographic images, endovascular revascularization technique and results, limb salvage and time to wound healing.

In this study, CKD was defined as a serum creatinine level greater than $1.5 \mathrm{mg} / \mathrm{dl}(\mathrm{GFR}<60)$ or as a history of chronic renal insufficiency requiring dialysis or kidney transplantation. Diabetes mellitus (DM) was defined as hemoglobin $\mathrm{A} 1 \mathrm{C}>6.5 \%$, fasting plasma glucose level > $126 \mathrm{mg} / \mathrm{dl}$ (7.0 mmol/l), or as hyperglycemic symptoms with a random plasma glucose level $>200 \mathrm{mg} / \mathrm{dl}$ (11.1 $\mathrm{mmol} / \mathrm{l})$. Arterial insufficiency was defined as an ankle/ brachial index (ABI) below 0.9 or greater than 1.3 (for patients with non-compressible arteries due to medial calcinosis), abnormal arterial waveforms or diminished pulse-volume recordings at the ankle. Technical success of endovascular intervention was defined as re-establishment of hind foot perfusion via direct in-line flow of at least 1 vessel with residual stenosis less than $30 \%$.

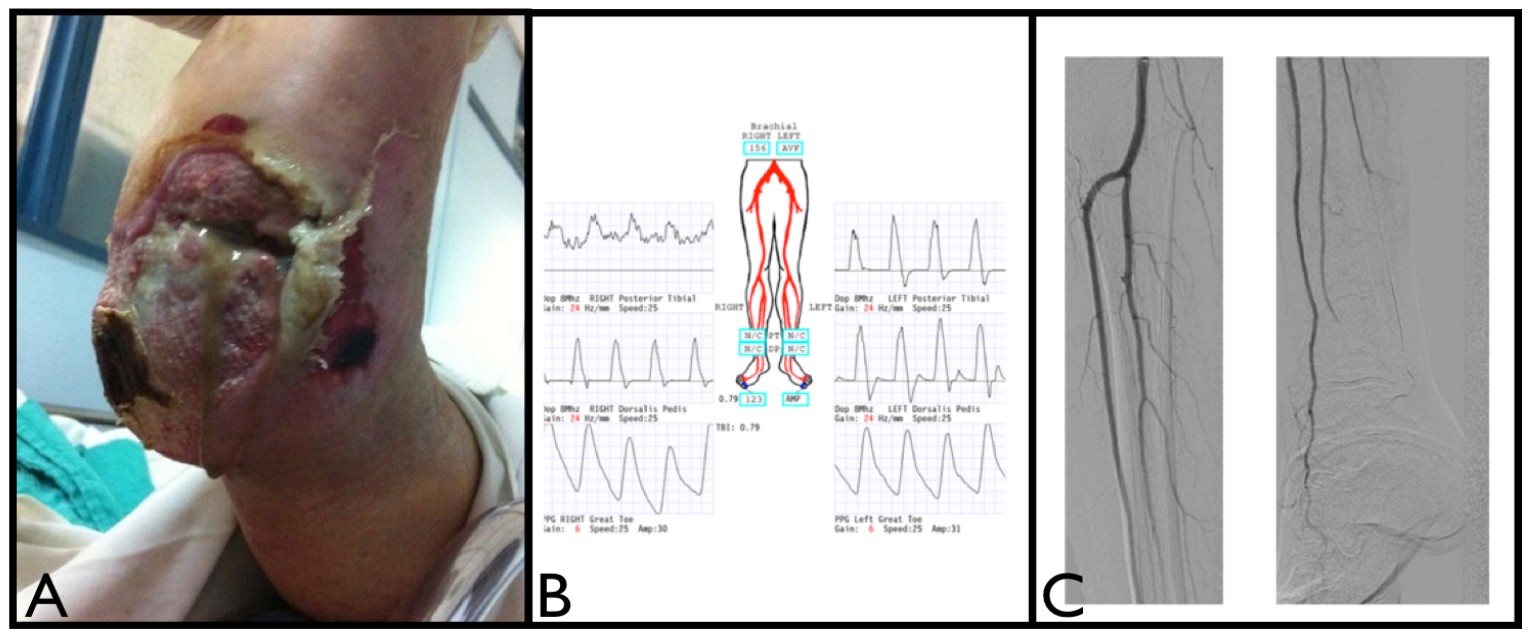

Figure 1: Clinical, non-invasive and invasive testing suggestive of an Orphan Heel Syndrome. A) Complex neuro-ischemic heel ulcer; B) Non-invasive Doppler waveform evaluation demonstrating normal Dorsalis Pedis waveforms with blunted Posterior Tibial waveforms; C) Angiography demonstrating normal forefoot perfusion with intact Anterior Tibial Artery and ischemic hind foot with occlusion of the Posterior Tibial Artery and Posterior Perforating branch of the Peroneal Artery. 


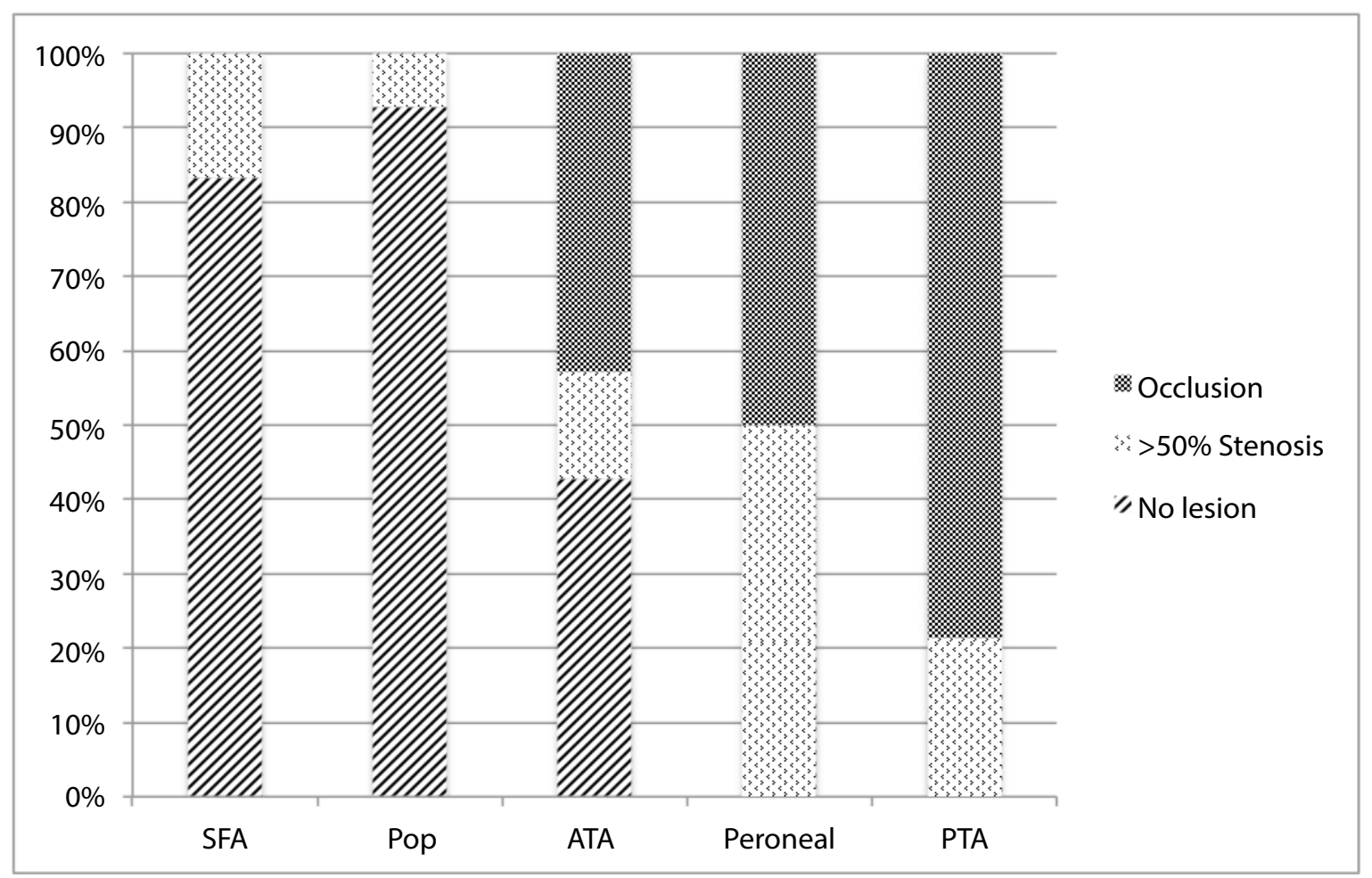

Figure 2: Pattern of disease in Orphan Heel Syndrome patients demonstrate Peroneal and Posterior Tibial Arteries predominance.

Patients were followed to their last clinic appointment visit or to the time of death. Descriptive statistics were used to report data in this analysis. Data were entered into a computerised spreadsheet (Microsoft Excel 2003, Microsoft corporation, Redmond, WA) and analysed using SPSS for $\mathrm{Mac}^{\circ}$, version 19.0 (SPSS, Chicago, IL).

\section{Results}

Over the 3-year study period, a total of 40 patients with 42 complex heel ulcers were identified. Of this initial group, $57 \%(n=24 / 42)$ underwent diagnostic angiography. After imaging, 12 patients (14 limbs) were conclusively identified as having typical anatomic findings of OHS. The mean age of this latter subset was $68.3 \pm$ 12.8 years, and $75 \%$ were male $(n=9 / 12)$. Comorbid conditions were common and significant. All patients exhibited long standing (mean time since diagnosis of $21 \pm 13.5$ years) poorly controlled diabetes with a mean hemoglobin A1C of $9.4 \pm 0.9 \mathrm{mg} / \mathrm{dl}$, and all patients had CKD with $75 \%(9 / 12)$ on dialysis. The mean serum albumin level was 2.8 (range 1.5-3.6) $\pm 0.4 \mathrm{~g} / \mathrm{dl}$.

Among the minority of patients in whom it was measurable, the mean $A B I$ was 0.53 (range 0.21-0.96); however, 8 patients $(67 \%)$ had non-compressible ankle arteries. Most of the limbs in the series $(9 / 14)$ had palpable Dorsalis Pedis pulse and none had Posterior Tibial pulse, as noted by a vascular surgeon in electronic medical record notes. Those who did have measurable systolic pressure at the dorsalis pedis level had a mean systolic pressure of $126.5 \pm 17.0 \mathrm{mmHg}$. On analysis of Doppler waveform patterns all 14 limbs had acceptable forefoot patterns with biphasic or pulsatile monophasic waveforms, 9 patients had blunted monophasic waveforms with little to non-diastolic progression and 5 of them had no posterior tibial waveform identifiable.

A total of 14 multilevel endovascular procedures were performed in this cohort. Diagnostic angiography revealed heterogenous distribution of hemodynamically significant occlusive disease in the Superficial Femoral Artery (36\%; 5/14 legs), popliteal (14\%; 2/14 legs) and anterior tibial artery (57\%; 8/14 legs). All patients in the data set had both peroneal and posterior tibial vaso-occlusive disease (defined as greater than $50 \%$ stenosis or occlusion) limiting the heel perfusion without any obvious collateral late filling of calcaneal branches after prolonged exposure digital subtraction (Figure 2). The mean lesion length of the peroneal and posterior tibial was $16.4 \pm 4.1 \mathrm{~cm}$. The therapeutic approach for all patients is summarized in Table 1.

All patients in the series had successful wire passage to either the Peroneal or the Posterior Tibial Arteries. After balloon angioplasty, technical success (defined earlier as in-line flow to one of these arteries without acute elastic recoil greater than $30 \%$ ) was accomplished in $78.6 \%$ (11/14). Two of the cases had failed passage of the PTA catheter, and one case had recoil greater than $30 \%$ after angioplasty-not candidate to stenting due to the extent of the lesion. Only 3 cases required concomitant proximal ilio-femoral inflow therapy, which was established using balloon angioplasty and self-expanding nitinol laser cut stents-at operators discretion. Most of the tibioperoneal therapy was accomplished by low profile $\left(0.018^{\prime \prime}\right.$ and/or 
Table 1: Distribution of endovascular therapy amongst the OHS subgroup.

\begin{tabular}{|l|l|}
\hline Patient & Procedure \\
\hline $\mathbf{1}$ & Balloon angioplasty of the PTA and ATA \\
\hline $\mathbf{2}$ & Balloon angioplasty of the PTA \\
\hline $\mathbf{3}$ & Unsuccessful angioplasty device delivery to Peroneal \\
\hline $\mathbf{4}$ & Balloon angioplasty of the Peroneal Artery \\
\hline $\mathbf{6}$ & Balloon angioplasty of the PTA \\
\hline $\mathbf{7}$ & Unsuccessful angioplasty device delivery to PTA \\
\hline $\mathbf{8}$ RLE & Balloon angioplasty of the PTA \\
\hline $\mathbf{8}$ LLE & Balloon/stent of EIA and SFA + balloon angioplasty of the Peroneal \\
\hline $\mathbf{9}$ & Angioplasty of the Popliteal Artery, unsuccessful PTA (elastic recoil) \\
\hline $\mathbf{1 0}$ & Balloon angioplasty of the PTA \\
\hline $\mathbf{1 1}$ RLE & Balloon/stent angioplasty of the Popliteal Artery + Angioplasty Peroneal \\
\hline $\mathbf{1 1}$ LLE & Angioplasty of the Popliteal and ATA and Peroneal \\
\hline $\mathbf{1 2}$ & Angioplasty of the SFA, Popliteal and PTA \\
\hline
\end{tabular}

EIA: External Iliac Artery; SFA: Superficial Femoral Artery; PTA: Posterior Tibial Artery; ATA: Anterior Tibial Artery.
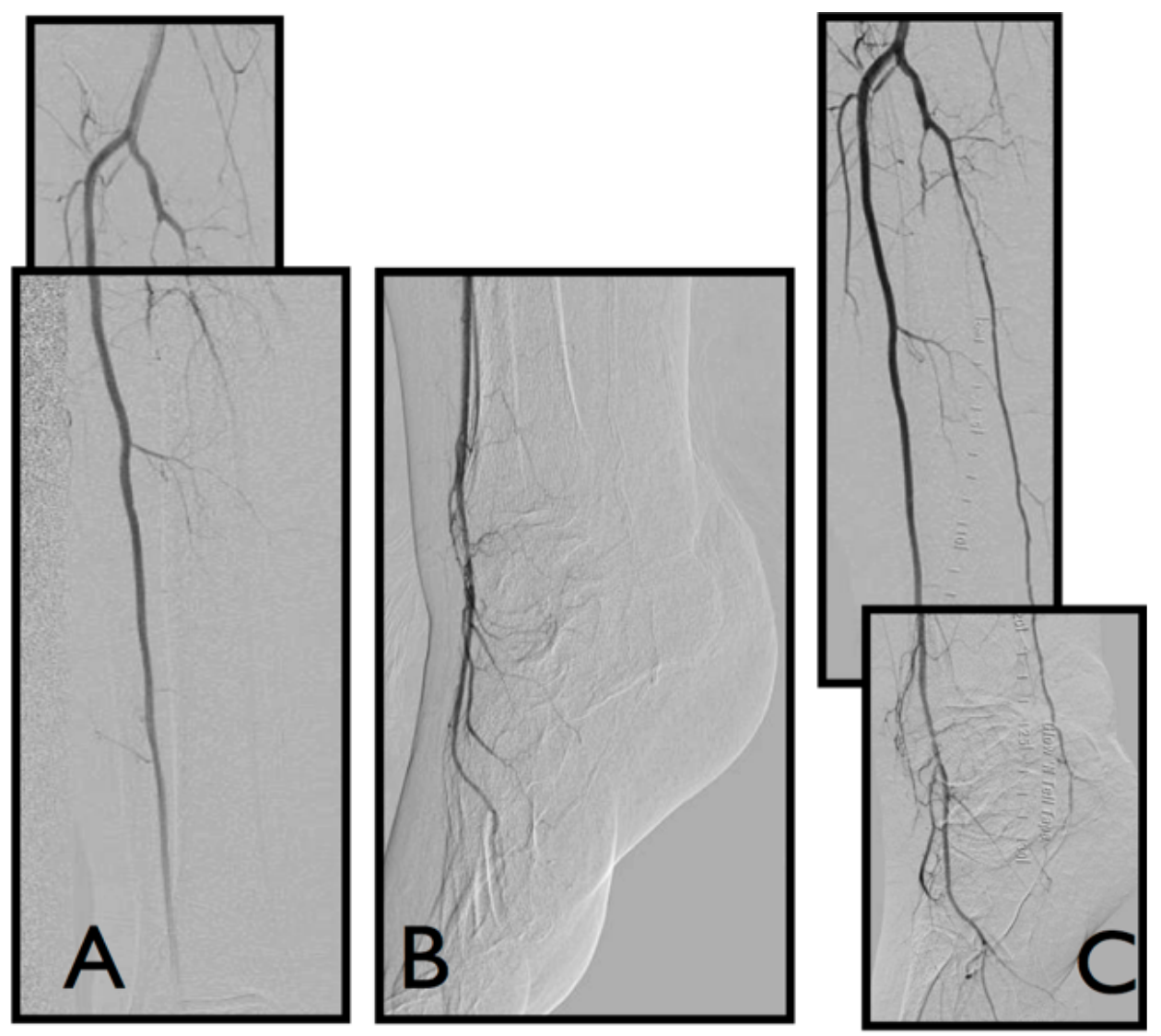

Figure 3: Successful endovascular intervention of an Orphan Heel Syndrome patient. A,B) Pre interventional image: Intact Anterior Tibial down to the foot with severely diseased Peroneal and Posterior Tibial Arteries; C) Post interventional image: Angiogram demonstrating in-line flow to the Posterior Tibial Artery status post successful endovascular therapy with balloon angioplasty.

0.014 ") balloon angioplasty without the need for stenting (Figure 3). Atherectomy was not used in this series. After endovascular intervention, the mean $A B I$ improved by 0.38 (range $0.27-0.79$ ) in those patients in whom it was measurable. All patients required wound debridement, 33.3\% (4/12) required tissue transfers and $16.7 \%$ $(2 / 12)$ received open surgical revascularization secondary to failed endovascular therapy.

As of June $2014,50 \%$ (7/14) of the OHS patients had healed, $22 \%$ (3/14) remained with intact index limbs but incompletely healed ulcers, $14 \%(2 / 14)$ had undergone below the knee amputation and $14 \%(2 / 14)$ were lost to follow up. The mean follow-up interval was 235 days (90-956 days). The range of times for complete wound healing was: $90,103,278,290,412,465$ and 956 days (mean healing time of $304 \pm 294$ days). Regarding those patients who specifically had technical success and adequate outpatient follow-up (with optimal wound care), the healing rate was $67 \%(6 / 9)$ with a mean time-tohealing of $364 \pm 321$ days. 


\section{Discussion}

The development of an ulcer over the calcaneus is a serious complication in patients with diabetes. When associated with PAD the prognosis is considered poor. Despite aggressive, multi-specialty team protocols, within 2 years nearly half of these patients will demonstrate progression of disease and require major limb amputation [13]. In the present study, a specific subgroup of heel ulcer patients with concomitant CKD, poorly diabetes and a specific anatomic pattern of regional foot ischemia were recognised, identified and characterised. For the purpose of this publication, the condition has been termed Orphan Heel Syndrome.

OHS patients had a mean hemoglobin A1C of $9.4 \pm$ $0.9 \mathrm{mg} / \mathrm{dl}$, mean serum albumin level of $2.8 \pm 0.4 \mathrm{~g} / \mathrm{dl}$ and $100 \%$ had renal failure, with $75 \%$ being dialysis-dependent. The latter combination of clinical findings has been demonstrated to be associated with such a high risk of limb loss that many authors advocate primary major amputation as the best alternative [12].

Although most patients $(57 \%, n=8 / 14)$ had non-compressible ankle arteries with unmeasurable ankle-brachial indexes, some had a measurable pressure at the dorsalis pedis level $(35 \%, n=5 / 14)$ with a mean systolic pressure of $126 \pm 17.0 \mathrm{mmHg}$. Moreover, $64 \%$ ( $n=9 / 14)$ of the OHS subset had readily palpable dorsalis pedis pulses. Based on the above and acceptable Anterior Tibial waveforms, most diagnostic guidelines would consider these patients to have a normal/acceptable foot perfusion.

The OHS patient population had absent posterior tibial pulses and blunted or absence of Doppler waveforms at this level. The disparity of fore and hind foot perfusion (based mainly on non-invasive waveform analysis and physical exam) supports the observation regarding vascular compartmentalization of the foot. The angiosome theory described by Taylor, et al. [14] has been extensively explained in other publication. This sub-group of patients, rather than defy the concept defines a subgroup of patients with intact forefoot angiosome perfusion, but ischemic calcaneal (medial/lateral) angiosome secondary to a series of clinical conditions that preclude angiosomal cross-coverage.

Although the angiosome theory has recently become a popular area of debate, few authors specifically address the 'choke vessel' or inter-angiosomic small caliber vascular bridges as having a role in the reason for this concept being crucial for dictating therapy (direct vs. indirect revascularization) $[14,15]$. These vascular bridges posses dynamic adaptability to redirect flow, which serves as the basis for maintenance of viable tissue in the presence of obstructive disease of the axial flow vessels.

Microvascular disease is an entity frequently described in patients with longstanding diabetes and advanced kidney failure [16]. The authors' hypothesis is that the clinical scenario of OHS patients negative-
Iy affects the luminal caliber and/or physiologic flow adaptability of the choke vessels hindering any potential angiosomal cross-coverage. Hence, the presence of peroneal, and to an even greater degree, posterior tibial occlusive disease, renders the heel an 'orphan' isolated from the perfusion of the rest of the foot. The heel can thus be mal-perfused even in the presence of a palpable dorsal pedal pulse and fully intact anterior tibial and dorsal pedal vessels. This hypothesis may well explain why in the published work from Azuma, et al. in relation to bypass surgery and target vessel selection, the healing rate for indirect angiosome revascularization was significantly slower than in the direct revascularization group, especially in the ESRD subgroup of patients [17].

Traditional noninvasive vascular testing falls short when it comes to assessing regional foot perfusion in the OHS subgroup of patients with heel ulcers. An assessment of whole foot perfusion based on forefoot perfusion (i.e. toe pressure) fails to detect hindfoot malperfusion. Faglia, et al. support perilesional transcutaneous oximetry of heel ulcers, as described in a recently published analysis of a solitary complex case that fits our much larger series of OHS [18]. Unfortunately, there are multiple well-known shortcomings of $\mathrm{TCPO}_{2}$, and our group favours research of newer techniques to assess regional perfusion such as indocyanine green angiography [19] or other alternatives such as bio-compatible implantable luminescent oxygen microsensors which due to their stable nature might even revolutionise surveillance protocols $[20,21]$.

In previous work, the authors have analysed data on angiographic disease patterns of heel ulcer patients and proposed a clinical classification scheme [7]: Grade - I patent posterior tibial, grade II - occluded posterior tibial reconstituted from peroneal, grade III - occluded posterior tibial reconstituted from dorsalis pedis, grade IV - no visible reconstitution of the posterior tibial and finally grade $\mathrm{V}$ - avascular heel. All of OHS patients had either class IV or $\mathrm{V}$ grade heel ischemia.

All patients were poor surgical candidates due to comorbid medical conditions, severe frailty, lack of adequate target arteries, insufficient vein conduit or a combination of these situations. As a result, endovascular management was initially attempted in all patients, which resulted in a $78.6 \%$ technical success rate. Two of the technical failures were secondary to failure to move the angioplasty catheter into place and one patient had significant acute recoil with suboptimal in line flow patterns to the heel. In the setting of severe medial calcinosis (classically expected in the CKD/advanced diabetes patient subgroup), technical success rate was found to be reasonable. Moreover, the fact that failure to achieve optimal flow was related to recoil or failure of device delivery, and not failure of wire passage; leaves room for improvement as technology for effective tibial intervention is being constantly miniaturised and new debulking tools are a work in progress. 
In general terms this specific subset of patients displays poor, extremely delayed wound healing. By the end of our review the ideal subgroup of patients with successful revascularization and adequate outpatient follow up resulted in a healing rate of $67 \%(6 / 9)$ and a mean time-to-heal of $364 \pm 321$ days. Additionally, two patients required a major below the knee amputation. Other authors describe positive predictors of healing as including normal renal function, palpable pedal pulses and patent posterior tibial arteries past the ankle [22]; unfortunately, all of these conditions are not shared by this particular subgroup. However, in view of our results, our group advocates aggressive revascularization attempts and optimal local management rather than a more radical stand of primary major amputation. Earlier recognition and revascularization when the wound is still small and not infected [23], may also lead to better outcomes. Moreover, due to the limited number of patients involved in this study, no interventional aspect of nonsurgical wound care showed greater efficacy than any other within the OHS subgroup.

\section{Conclusion}

Orphan heel syndrome is a condition characterised by the triad of heel ulceration, diabetic end stage renal disease, and an avascular heel due to compartmentalised foot circulation with an isolated heel due to posterior tibial and peroneal occlusive disease, pedal arch disease, and most likely disease progression into choke vessels. In this specific cohort of patients, classic noninvasive vascular testing often fails to detect ischemia, warranting a higher index of suspicion and novel techniques to evaluate topographical foot perfusion. Incorporation of endovascular therapy into management protocols results in acceptable limb salvage and wound healing rates. It is the author's opinion that OHS is not recognised until a wound has reached a late stage of development, delaying attempts at timely intervention. Many patients with heel ulcer and palpable Dorsalis Pedis pulses or normal ABIs never trigger a specialist consultation. Awareness of OHS amongst primary care doctors, nephrologists and other specialists who deal with vascular patients should be raised in the hope that greater familiarity might lead to focused preventive efforts, earlier recognition and more prompt referral to a vascular and endovascular surgery service.

\section{Author Contributions}

MM, JMiller researched data. MM, JMills, JMiller wrote manuscript. KG, JMills, DA. BB reviewed/edited manuscript.

\section{Note}

This research was previously presented at the $28^{\text {th }}$ annual meeting of the Western Vascular Society as a full presentation on September $24^{\text {th }}, 2013$.

\section{References}

1. Brem $\mathrm{H}$, Jacobs $\mathrm{T}$, Vileikyte $\mathrm{L}$, Weinberger $\mathrm{S}$, Gibber $\mathrm{M}$, et al. (2003) Wound-healing protocols for diabetic foot and pressure ulcers. Surg Technol Int 11: 85-92.
2. Saqib NU, Domenick N, Cho JS, Marone L, Leers S, et al. (2013) Predictors and outcomes of restenosis following tibial artery endovascular interventions for critical limb ischemia. J Vasc Surg 57: 692-699.

3. Ndip A, Rutter MK, Vileikyte L, Vardhan A, Asari A, et al. (2010) Dialysis treatment is an independent risk factor for foot ulceration in patients with diabetes and stage 4 or 5 chronic kidney disease. Diabetes Care 33: 1811-1816.

4. Ali R, Qureshi A, Yaqoob MY, Shakil M (2008) Total contact cast for neuropathic diabetic foot ulcers. J Coll Physicians Surg Pak 18: 695-698.

5. Isenberg JS, Costigan WM, Thordarson DB (1995) Subtotal calcanectomy for osteomyelitis of the os calcis: A reasonable alternative to free tissue transfer. Ann Plast Surg 35: 660-663.

6. Gooden MA, Gentile AT, Mills JL, Berman SS, Demas CP, et al. (1997) Free tissue transfer to extend the limits of limb salvage for lower extremity tissue loss. Am J Surg 174: 644-648.

7. Gentile AT, Berman SS, Reinke KR, Demas CP, Ihnat DH, et al. (1998) A regional pedal ischemia scoring system for decision analysis in patients with heel ulceration. Am J Surg 176: $109-114$.

8. Karp NS, Kasabian AK, Siebert JW, Eidelman Y, Colen S (1994) Microvascular free-flap salvage of the diabetic foot: A 5-year experience. Plast Reconstr Surg 94: 834-840.

9. Lantis JC $2^{\text {nd }}$, Conte MS, Belkin M, Whittemore AD, Mannick JA, et al. (2001) Infrainguinal bypass grafting in patients with end-stage renal disease: Improving outcomes? J Vasc Surg 33: 1171-1178.

10. Edwards JM, Taylor LMJ, Porter JM (1988) Limb salvage in end-stage renal disease (ESRD). Comparison of modern results in patients with and without ESRD. Arch Surg 123: 1164-1168.

11. Kabra A, Suresh KR, Vivekanand V, Vishnu M, Sumanth $R$, et al. (2013) Outcomes of angiosome and non-angiosome targeted revascularization in critical lower limb ischemia. J Vasc Surg 57: 44-49.

12. Dosluoglu HH, Attuwaybi B, Cherr GS, Harris LM, Dryjski ML (2007) The management of ischemic heel ulcers and gangrene in the endovascular era. Am J Surg 194: 600-605.

13. Klamer TW, Towne JB, Bandyk DF, Bonner MJ (1987) The influence of sepsis and ischemia on the natural history of the diabetic foot. Am Surg 53: 490-494.

14. Taylor GI, Pan WR (1998) Angiosomes of the leg: Anatomic study and clinical implications. Plast Reconstr Surg 102: 599-616.

15. Alexandrescu V, Söderström M, Venermo M (2012) Angiosome theory: Fact or fiction? Scand J Surg 101: 125-131.

16. Bejan-Angoulvant $T$, Bergerot $C$, Juillard $L$, Mezergues $A$, Morelon E, et al. (2012) Myocardial microvascular disease and major adverse cardiovascular events in patients with end-stage renal disease: Rationale and design of the MICROCARD study. Nephrol Dial Transplant 27: 2886-2891.

17. Azuma N, Uchida H, Kokubo T, Koya A, Akasaka N, et al. (2012) Factors influencing wound healing of critical ischaemic foot after bypass surgery: Is the angiosome important in selecting bypass target artery? Eur J Vasc Endovasc Surg 43: 322-328.

18. Faglia E, Clerici G, Caminiti M, Vincenzo C, Cetta F (2013) Heel ulcer and blood flow: The importance of the angiosome concept. Int J Low Extrem Wounds 12: 226-230. 
19. Braun JD, Trinidad-Hernandez M, Perry D, Armstrong DG, Mills JL (2013) Early quantitative evaluation of indocyanine green angiography in patients with critical limb ischemia. $J$ Vasc Surg 57: 1213-1218.

20. Lee YE, Ulbrich EE, Kim G, Hah H, Strollo C, et al. (2010) Near infrared luminescent oxygen nanosensors with nanoparticle matrix tailored sensitivity. Anal Chem 82: 8446-8455.

21. Montero-Baker M, Morelli-Alvarez L, Helton K, Au-Yeung KY (2014) The use of Micro-Oxygen Sensors (MOXYs) to determine dynamic relative oxygen indices in the foot of patients with Critical Limb Ischemia (CLI) during an endovas- cular therapy: The first-in-man 'Si Se Puede' study. Journal of Vascular Surgery 60: 548-549.

22. Treiman GS, Oderich GS, Ashrafi A, Schneider PA (2000) Management of ischemic heel ulceration and gangrene: An evaluation of factors associated with successful healing. $J$ Vasc Surg 31: 1110-1118.

23. Mills JL Sr, Conte MS, Armstrong DG, Pomposelli FB, Schanzer A, et al. (2014) The society for vascular surgery lower extremity threatened limb classification system: Risk stratification based on wound, ischemia, and foot infection (WIfl). J Vasc Surg 59: 220-222. 